\title{
Die sozial-unternehmerische Stadt: Gesellschaftliche Ungleichheit, Reproduktionskrise und Stadtpolitik in Hamburg
}

\author{
Jan Kemper and Anne Vogelpohl \\ Department Soziale Arbeit, Hochschule für Angewandte Wissenschaften Hamburg, Hamburg, Germany \\ Correspondence: Jan Kemper (jan.kemper@dataplebs.de)
}

Received: 20 May 2019 - Revised: 11 May 2020 - Accepted: 2 June 2020 - Published: 17 July 2020

\begin{abstract}
Kurzfassung. Entrepreneurial and social urban policies contradictorily depend from each other: growth policies aiming to increase investment and population numbers produce a crisis of reproduction (i.e. housing or care); and social policies aiming to resolve this crisis allegedly require entrepreneurial policies as fuel for tax resources financing social goals. We investigate this interrelationship along the recent housing and childcare policies in the city of Hamburg, Germany. We show that as long as growth and competition are primary objectives of urban governments, a compensatory social policy is needed to legitimate the entrepreneurial axiom - and therefore has a specific character: social policy in the social-entrepreneurial city is actually addressing inequalities, but it eventually is aimed at all classes, secures capital accumulation and is gendered. This paper contributes to understanding urban crises of reproduction by exposing that current social policies aiming to solve this crisis merely consolidate the crisis-prone growth policies.
\end{abstract}

\section{Einleitung}

Sozialpolitische Interventionen werden in den letzten Jahren wieder verstärkt, u.a. weil die sozialen Folgen neoliberaler Tendenzen nach der großen Finanz- und Wirtschaftskrise von 2008 vermehrt sichtbar geworden sind. Die Covid19-Krise 2020 zeigt den Bedarf an staatlichen Eingriffen in Extremform. Auf städtischer Ebene lassen sich z.B. in der Wohn- und Kinderbetreuungspolitik Interventionen erkennen, die zugleich den Druck auf die Lebensverhältnisse städtischer privater Haushalte verringern und Akzeptanzverlusten einer Politik der unternehmerischen Stadt entgegenwirken sollen. Wir möchten diesen Zusammenhang in den Blick nehmen und ihn als ,sozial-unternehmerische Stadt" konzeptualisieren. Deren Kern ist das Zusammenspiel der sozialpolitischen Bewältigung sozialer Problemlagen mit der Verstetigung der ökonomischen Wettbewerbsund Wachstumsstrategie der unternehmerischen Stadt. Die Absicherung der unternehmerischen Stadt braucht eine soziale Stadtpolitik - so die Perspektive unseres Beitrages.

Am Beispiel der Neujustierungen in den stadtpolitischen Feldern „Wohnen“ und „Kinder betreuen“ in Hamburg möchten wir die These der Formierung einer sozialunternehmerischen Stadt plausibilisieren. Um diese noch differenzierter beschreiben zu können, wäre es erstrebenswert, die engen Verwebungen von Sozial- und Wirtschaftspolitik auch in anderen Feldern der Reproduktion nachzuvollziehen (Pflege, Bildung, Gesundheit - um nur einige zu nennen). Hier vertiefen wir jedoch vorerst die bestehende Debatte um soziale Ungleichheiten und Stadt. An der „Rückkehr der Wohnungsfrage" (Schönig et al., 2015) wird bereits seit Längerem debattiert, inwieweit sich in aktuellen wohn- und stadtpolitischen Gestaltungsabsichten gesamtgesellschaftliche Wandlungsprozesse abzeichnen, die als Bruch mit den Konfigurationen der neoliberalen unternehmerischen Stadt zugunsten einer post-neoliberalen Phase der Stadtentwicklung gedeutet werden können (Holm, 2017; Metzger und Schipper, 2017; Rinn, 2018; Vogelpohl und Buchholz, 2017; Vollmer und Kadi, 2018). Rinn (2018:22) spricht bereits ebenfalls von einer „unternehmerisch-integrierte[n] Stadtentwicklungspolitik“ und stellt fest, dass ,soziale wie unternehmerische Stadtentwicklungsstrategien konvergieren und tendenziell ununterscheidbar werden“. Wir möchten nun 
zuspitzen: Die Politiken sind nicht ununterscheidbar, sondern auf spezifische Weise voneinander abhängig.

Die Debatte um Wohnungspolitik als Gradmesser gesamtgesellschaftlicher Entwicklungen kommt nicht von ungefähr. An der Wohnungsfrage lässt sich ablesen, dass und wie mit politischen Neujustierungen auf die krisenhaft erlebte Instabilität der unternehmerischen Stadt sowie auf die sich dagegen richtenden Proteste reagiert worden ist. Mit der Rückkehr der Wohnungsfrage werden daher zum einen gesellschaftspolitische Grenzen der bisherigen unternehmerischen Stadtentwicklungspolitik offensichtlich. Zum anderen stellen sich mit ihr (erneut) die Fragen einer kritischen Politik- und Staatsanalyse, wie sie Eingang auch in Debatten der Geographie gefunden haben - so etwa nach der sozialen Wirkmächtigkeit, der sozialen Selektivität und nach der gesellschaftspolitischen Funktion (lokal-)staatlicher Interventionen oder nach den Wirkungen, Möglichkeiten und Beschränkungen gesellschaftlicher Reformstrategien.

Die Thematisierung der Politik der Wohnungsversorgung aktualisiert aber nicht nur die Problemperspektiven einer kritischen Analyse politisch-staatlicher Intervention. Sie drängt auch auf eine empirische Erweiterung des Aufmerksamkeitsspektrums. „Wohnungspolitik ist nur ein Feld der Stadtpolitik“", schränken etwa Vollmer und Kadi (2018:262) die Verallgemeinerungsfähigkeit ihres Untersuchungsergebnisses einer ,(vorläufige[n]) punktuelle[n] Abkehr vom neoliberalen Paradigma" ein. Ob ein (lokal-)staatliches Krisenmanagement auch in Reaktion auf andere aktuelle urbane Protest- und Problemfelder - wie etwa die „Kitakrise“ oder der „Pflegenotstand“ - greift und worauf es hindeutet, ist demnach empirisch noch nicht ausgemacht. Deshalb gehen wir daran, den Gegenstandsbereich der Stadtforschung empirisch auszuweiten: Wir verknüpfen Beobachtungen zu Neujustierungen in der Politik der Wohnungsversorgung mit solchen im Feld der Kinderbetreuung.

Konzeptionelle Bindeglieder für diese Verknüpfung geben uns die in Abschnitt 2 erörterten Theoriestränge der politisch-ökonomischen und der feministischen Stadtforschung. Sie dechiffrieren stadtpolitische Problemfelder und ihre städtisch-staatliche Bearbeitung vor allem als solche der Reproduktionssphäre kapitalistischer Gesellschaften und deren Regulation. Mit ihnen lässt sich zudem das Grundproblem jedes (wohlfahrts-)staatlichen Krisenmanagements im Kapitalismus erörtern, nämlich dass die finanziellen Ressourcen für sozialpolitische Interventionen eben aus jenen ökonomischen Prozessen geschöpft werden müssen, die selbst regelmäßig den Ausgangspunkt sozialer Problemlagen bilden. Mit anderen, für die städtische Ebene reformulierten Worten: Nur ein weiterer Erfolg in der Konkurrenz der Städte um ökonomisches Kapital und einkommensstarke soziale Schichten kann gegenwärtig offenbar die ökonomische Grundlage für eine sozialpolitische Agenda geben, die überhaupt erst wegen dieser hegemonialen Konkurrenz verstärkt nötig ist.
Die These der Transformation der unternehmerischen in die sozial-unternehmerische Stadt plausibilisieren wir in Abschnitt 3 entlang politischer Regulationen der Reproduktionssphäre im Stadtstaat Hamburg. In Hamburg hat sich im Städtevergleich relativ früh (nämlich mit dem erfolgreichen Versuch der Hamburger Sozialdemokratie 2011, mit dem Versprechen sozialpolitischer Neuerungen die Regierungsmacht zurückzuerlangen) eine stadtpolitische Programmatik durchsetzen können, die verspricht, in zentralen Bereichen der Alltagsorganisation private Haushalte von $\mathrm{zu}$ hohen Lebenshaltungskosten zu entlasten, Prekarisierungs-, Polarisierungs- und Segregationstendenzen zu minimieren sowie Partizipations- und Mitbestimmungswünsche zu respektieren. Diese Programmatik wurde und wird öffentlich als Renaissance einer sozialen Stadtpolitik kommuniziert. In der sozialwissenschaftlichen Stadtforschung ist sie als eine Reaktion auf die politisch-sozialen Folgeprobleme einer Neoliberalisierung der Städte sowie auf den von städtischen sozialen Bewegungen ausgeübten politischen Druck auf eine veränderte, post-neoliberale Stadtpolitik hin ausgedeutet worden (vgl. Rinn, 2016; Vogelpohl und Buchholz, 2017).

Zentrale Interventionsfelder dieser politischen Neuorientierung waren und sind die Reproduktionsbereiche „Wohnen“ und „Kinder betreuen“. An den dort zum Tragen kommenden Ansätzen, den Ansätzen (1) zur Schaffung von mehr Wohnraum und von bezahlbaren Wohnungen sowie (2) der Einführung einer kostenlosen Grundbetreuung für Kinder bis zur Einschulung, verfolgen wir in diesem Beitrag, wie die sozialdemokratisch geführte Stadtregierung Hamburgs politische Regulationen der Reproduktion durchsetzt. Sie wirken ebenso als Maßnahmen für einen sozialen Ausgleich, die einer übergeordneten städtischen Wachstumspolitik soziale Akzeptanz und politische Legitimität sichern, wie sie als Maßnahmen für eine ökonomische Stimulation gelten können, die Wachstum und Wettbewerbsfähigkeit begünstigen.

Die sich aus unserer Untersuchung ergebenden Konturen sozial-unternehmerischer Stadtpolitik bilanzieren wir in Abschnitt 4.

\section{Wohnungsfrage, Wohlfahrtsstaat und women*'s work}

Krisendiagnosen über die moderne Gesellschaft und Aussagen zur Stadtentwicklung verweisen aufeinander. Seit Beginn des Doppelprozesses von Industrialisierung und Urbanisierung geben Untersuchungen der Lebensverhältnisse in den großen Städten Auskunft über die konflikt- und krisenvermittelten politisch-ökonomischen Regelmäßigkeiten moderner Vergesellschaftung. Die so gewonnenen Erkenntnishorizonte wiederum formten zugleich die Problemperspektiven, mit denen die verschiedenen sozialen Lebensbedingungen und -chancen in den Städten erst untersuchbar wurden. 
Kaum verwunderlich also, wenn in der gegenwärtigen Thematisierung einer „Rückkehr der Wohnungsfrage“ semantisch und inhaltlich mit Friedrich Engels (1820-1895) an einen Klassiker einer ebenso empirisch fundierten wie gesellschaftstheoretisch orientierten Sozialwissenschaft angeknüpft wird. Engels' in den Jahren 1872/73 veröffentlichten Beiträge Zur Wohnungsfrage (Engels, 1984) erinnern nicht nur qua Titel daran, dass mit der heutigen Wohnungsfrage offensichtlich ein Dauerproblem reaktualisiert worden ist. Auch ein Blick in seine damals vorgebrachte Argumentation lässt nach wie vor Nützliches für eine Analyse der Wohnungsversorgung in den Koordinaten von "Markt“ und „Staat" deutlich werden. Zugleich weist sie deutlich darüber hinaus. Engels thematisiert die Wohnungsfrage in einem Problemzusammenhang mit systemischen Aspekten der kapitalistischen Klassengesellschaft. Er macht damit die damaligen Ansätze zur Integration der Arbeiterklasse in die gegebene soziale Ordnung und somit sozialpolitische Versuche der Regulation des Klassenkonflikts zum Gegenstand seiner Analyse und Kritik.

Um Verbindungspunkte zwischen stadtpolitischen Regulationen der Felder „Wohnen“ und „Kinder betreuen“ sowie eine gemeinsame Perspektive darauf zu gewinnen, folgen wir zunächst der in Zur Wohnungsfrage (Engels, 1984) gelegten Spur der Analyse. Entlang der dort aufscheinenden Problematik eines sozialpolitisch stabilisierten Kapitalismus arbeiten wir einen Bezugsrahmen für die Analyse und Kritik staatlicher Sozialpolitik heraus (Abschnitt 2.1). Anschließend vertiefen wir die Diskussion staatlicher Sozialpolitik mit einer Erinnerung an die sozialwissenschaftliche Analyse des „Doppelcharakters des Wohlfahrtsstaats" und an das stadtsoziologische Konzept der „kollektiven Konsumtion“. Damit lässt sich der analytische Blick für die Krisenregulationen des lokalen Staats und für eine empirische Untersuchung der tatsächlichen Effekte städtisch-staatlicher Sozialpolitiken auf verschiedene Dimensionen gesellschaftlicher Ungleichheit öffnen (Abschnitt 2.2). Diese Öffnung schließlich verfolgen wir an der feminisierten Reproduktionsarbeit und ihrer städtischstaatlichen Regulation. An diesem Verhältnis arbeiten wir die Bedeutung der Krise der unternehmerischen Stadt als Krise der Reproduktion und einer darauf antwortenden urbanen Regulation der Reproduktion für die Formierung einer sozial-unternehmerischen Stadt heraus (Abschnitt 2.3).

\subsection{Das Reproduktionsniveau der Arbeiterklasse und der klassenübergreifende Charakter der Wohnungsfrage}

Die Basisargumentation in Zur Wohnungsfrage (Engels, 1984) ist so einfach wie grundsätzlich. Sie bestimmt - systematisch betrachtet - den kapitalistischen Produktionsprozess als einen Ausbeutungsprozess, der auf der Erzeugung und sozial selektiven Aneignung von Mehrwert beruht (Engels, 1984:8). Mit dieser Grundposition tritt Engels an den damaligen Krisendiskurs über die „Wohnungsnoth“ (Engels, 1984:7) bzw. über die „Miethsnoth“ (Engels, 1984:11) heran. Er greift dabei zwar auch die „schlechten Wohnverhältnisse der Arbeiter“ auf, spricht ihre „eigenthümliche Verschärfung“ (Engels, 1984:7) an und geht auf Gründe für sie ein. Doch eine Beschreibung und Erklärung der Wohnverhältnisse der armen, besitzlosen arbeitenden Klasse steht nicht im Vordergrund dieser Streitschrift. Stattdessen sind Engels' Ausführungen zur Wohnungsfrage vor allem gegen zeitgenössische sozialpolitische Vorschläge gerichtet, die auf eine Bewältigung der proletarischen Wohnungsmisere zielen. Seine Polemiken verfolgen das Ziel, herauszustellen, dass sozialpolitische Eingriffe innerhalb der Gesetzmäßigkeiten der kapitalistischen Produktionsweise untauglich sind, das proletarische Klassenschicksal aufzulösen.

Dafür zieht Engels zunächst einmal einen deutlichen Trennungsstrich zwischen der Relation Mieter-Vermieter einerseits, der Beziehung Arbeiter-Kapitalist andererseits. In der Beziehung Mieter-Vermieter vermittelt sich demnach ein „einfacher Waarenverkauf“ (Engels, 1984:11). In diesem ,ganz gewöhnlichem Waarengeschäft zwischen zwei Bürgern“ (Engels, 1984:12) tritt „,der Miether - selbst wenn er Arbeiter ist - [...] als vermögender Mann auf" (Engels, 1984:11-12), um seinem Gegenüber „den zeitweiligen Gebrauch einer Wohnung“ (Engels, 1984:11) abzukaufen. Sofern sich bei diesem Geschäft die Mietpartei übervorteilt fühlt und einen ihr unangemessen erscheinenden hohen Preis für den Wohnungsgebrauch zahlt, darf sie sich als Opfer handelsüblicher „Prellerei“ (Engels, 1984:11) sehen. Der Kauf bzw. Verkauf von Arbeitskraft gegen Lohn dagegen unterscheidet sich von solchen ,ganz gewöhnlichen Waarengeschäften" und ihren Unbequemlichkeiten in dem einen, aber alles entscheidenden Punkt, ,,daß unsere jetzige Gesellschaftsordnung den Kapitalisten in den Stand setzt, die Arbeitskraft des Arbeiters zu ihrem Werth zu kaufen, aber weit mehr als ihren Werth aus ihr herauszuschlagen, indem er den Arbeiter länger arbeiten läßt, als zur Wiedererzeugung des für die Arbeitskraft gezahlten Preises nöthig ist“" (Engels, 1984:8).

Mit dem Hinweis auf die Warenförmigkeit der Arbeitskraft eröffnet sich Engels den Raum für eine Diskussion der Folgen, die sozialpolitische Eingriffe in das Geschäft mit der Ware Wohnen mit sich bringen würden. Ermöglichen diese Interventionen ein Sinken der Wohnkosten, gleicht das nach Engels (1984:36f) einem Absenken der Kosten für die Reproduktion der als Ware gehandelten, für den Einsatz im Produktionsprozess gekauften und dort verausgabten Arbeitskraft. Weil sich aber der Wert der Arbeitskraft wiederum an den Kosten ihrer Reproduktion orientiert, laufen günstigere Wohnkosten ,wie jede dauernde Preiserniedrigung der Lebensbedürfnisse des Arbeiters" für Engels (1984:36) „schließlich“ auf eine Verbilligung des Werts der Arbeitskraft, daher auf eine Senkung des Arbeitslohns und somit auf eine Steigerung der Mehrwertrate hinaus. Was sich daher vielleicht kurzfristig und auf 
der Ebene der einzelnen proletarischen Haushalte als eine Besserung der Lebensverhältnisse darstellt, wird sich daher mittelfristig und in klassenanalytischer Perspektive bestenfalls als ein Nullsummenspiel, im schlechtesten Fall als ein Angriff auf ein gegebenes Reproduktionsniveau der Arbeiterklasse erweisen.

Mit diesen Einordnungen weist Engels nicht nur zeitgenössische sozialpolitische Vorschläge zur Lösung der Wohnungsfrage als vergeblich, wenn nicht gar kontraproduktiv für eine Verbesserung der proletarischen Lebensverhältnisse zurück. Sein Szenario erlaubt ihm umgekehrt auch, das gesellschaftspolitische Aufwerfen der Wohnungsfrage selbst einzuordnen, also Hinweise zu einer gesellschaftlichen Problemanerkennung und -durchsetzung zu geben. Die Wohnungsmisere seiner Zeit macht nach Engels (1984:8) „,nur so viel von sich reden, weil sie sich nicht auf die Arbeiterklasse beschränkt, sondern auch das Kleinbürgerthum mit betroffen hat." Erstere sei zwar „sicher härter als jede wohlhabendere Klasse“ (Engels, 1984:8) von der Wohnungsnot gezeichnet, diese aber bilde gerade keinen ,ausschließlich auf die Arbeiterklasse drückenden Uebelstand“ (Engels, 1984:11). Vielmehr lässt der klassenübergreifende Charakter der als Wohnungsfrage thematisierten Problemlagen diese erst gesellschaftspolitisch relevant werden. Gerade weil sie mehr ist als allein ein Problem der armen, besitzlosen und deshalb ihre Arbeitskraft verkaufenden Klasse, ist die Wohnungsfrage Gegenstand sozialpolitischer Sorgen und Programme des ,kleinbürgerlichen Sozialismus" einerseits, der Integrationsversuche der Herrschaftsformen des „Bonapartismus“ andererseits geworden.

Solche Ideologien und Praktiken der Integration hat Engels in Zur Wohnungsfrage (Engels, 1984) vor Augen und zum Gegner. Sie sind ihm zufolge darauf gerichtet, vor dem Hintergrund der Klassenstruktur und den Klassenauseinandersetzungen der kapitalistischen Gesellschaft (und abgestützt vor allem auf die kleinbürgerlichen, ,mittleren“ Klassen) eine Gesellschaftspolitik zu entfalten, die mittels punktuellen Reformen und „Staatshilfe“ (Engels, 1984:49), eben etwa auf dem Gebiet des Wohnens, klassenübergreifend soziale Notlagen abmildert und somit auf breiter sozialer Basis ruhende Loyalitätsbezüge sichert. Gerade darüber werden die fundamentalen kapitalistischen Produktions-, Verkehrs- und Verteilungsverhältnisse stabilisiert. Dagegen war Engels motiviert $\mathrm{zu}$ verdeutlichen, dass nicht eine sozialreformerische Behandlung der Wohnungsfrage die Armen, Besitzlosen und deshalb Arbeitenden von diesen Attributen befreien, sondern umgekehrt allein eine sozialrevolutionäre Aufhebung der kapitalistischen Klassenstruktur und -beziehungen auch solche ,sekundären Uebelstände“ (Engels, 1984:8) wie die Wohnungsnot auflösen wird.

\subsection{Der Doppelcharakter des Wohlfahrtsstaats und das Konzept der kollektiven Konsumtion}

Die bei Engels (1984) angedeutete Erfahrung einer sozialpolitischen Stabilisierung des sozialen Systems hat ebenso wie die dort anklingende Thematisierung klassenübergreifender, tendenziell quer zur Auseinandersetzung zwischen den Klassen liegender Konfliktlinien eine Ausarbeitung in neo-marxistisch orientierten Theoremen der 1970er Jahre über den massendemokratisch abgestützten und wohlfahrtsstaatlich regulierten Spätkapitalismus gefunden (etwa Habermas, 1973; O’Connor, 1974; Offe, 1972). Was sich bei Engels (1984) vorsichtig andeutet, wird dort in Überlegungen zum ,dualen und widersprüchlichen Charakter des kapitalistischen Staats“ (O’Connor, 1974:17) sowie in der Betonung neuer, horizontaler Disparitäten systematisch fortgeführt.

Analysen zur Rolle und Bedeutung des spätkapitalistischen Staates betonen dessen Doppelcharakter. Demnach hat der Staat zum einen die Funktion, über umfassende Interventionen in Wirtschaft und Gesellschaft den Verwertungsprozess des Kapitals zu gewährleisten, also „die Bedingungen zu erhalten oder $\mathrm{zu}$ schaffen, in denen eine gewinnträchtige Kapitalakkumulation möglich ist" (O'Connor, 1974:16). Zum anderen ist er darauf hin orientiert, ,soziale Harmonie zu erhalten oder zu schaffen“(O'Connor, 1974:16) und somit Massenloyalität zu garantieren. Beide Aufgaben bzw. Funktionen des Staates, die von „Akkumulation“ und „Legitimation“, bedingen einander bzw. sind widersprüchlich aufeinander verwiesen: In der Verfolgung von Akkumulation wird Legitimation nötig, die Garantie von Legitimation aber ist abhängig von und begrenzt durch die Ressourcen aus dem Wirtschaftsbzw. Akkumulationsprozess (vgl. O’Connor, 1974:16).

Die Impulse für diese doppelte, konfliktbehaftete Aufgabenstellung des Staates werden, ganz allgemein zusammengefasst, in der systemischen Instabilität der kapitalistischen Produktionsweise verortet. Deren prinzipiell krisen- und konfliktvermittelte Funktionsweise untergräbt tendenziell die eigenen Bestandsvoraussetzungen. Der gesellschaftliche Produktions- und kapitale Verwertungsprozess ist demnach als latent gefährdet zu begreifen. In dem Maße aber, wie das ökonomische System selbst die „Reproduktion der Produktionsbedingungen“" (Althusser, 2012:82) unterläuft, sichern politisch-administrative Eingriffe sein Funktionieren ab. Diese Interventionen wiederum können sich in demokratisch verfassten Gesellschaften nun nicht allein auf die (Wieder-)Herstellung der systemischen Voraussetzungen für ein wirtschaftliches Wachstum beschränken, sondern müssen auch sozialen Frieden und politische Legitimation gewährleisten.

In Untersuchungen des lokalen Staates bzw. von Stadtpolitik wurde dieser allgemeine Deutungsrahmen am Prozess der kollektiven Konsumtion operationalisiert (vgl. Castells, 1977; O’Connor, 1974; Offe, 1972:153-168). Mit 
ihm sind die „kollektiven Versorgungsleistungen“ (Offe, 1972:157) der Städte angesprochen. Mit diesen wurde vor dem Hintergrund gestiegener Anforderungen an die Produktivität der Arbeitskraft als auch der erweiterten Reproduktionsbedürfnisse der arbeitenden Klasse daran gegangen, einen Teil der Reproduktionskosten der Arbeitskraft zu vergesellschaften. Über den städtisch-staatlichen Bau und den Unterhalt von Wohnungen, Gesundheitseinrichtungen, Transport- und Kommunikationsnetzen, Kindertages- und Ausbildungsstätten etc. sind, so die damalige Einschätzung, das Reproduktionsniveau angehoben und somit Loyalitätsressourcen aufgebaut, zugleich das Lohnniveau eingefroren und somit die fortlaufende Akkumulation des Kapitals garantiert worden.

Zugleich aber ließ sich entlang der Vergesellschaftung der Reproduktionskosten der Arbeitskraft hervorheben, dass eine urbane Politik der kollektiven Konsumtion ungleiche Lebensverhältnisse nicht nur $\mathrm{zu}$ vermindern, sondern soziale Verteilungskonflikte auch zu reproduzieren, zu verstärken oder gar neue Spaltungslinien zu produzieren vermag. Denn eine zunehmend politisch-administrative Organisation von Reproduktionsbedingungen, so etwa Offe (1972:157ff), verschiebt die Auseinandersetzungen um soziale Lebensverhältnisse weg von dem in den Betrieben ausgetragenen „Lohnkämpfen“ (Offe, 1972:159) hin zu Aktionen, die von Bürgerinitiativen getragen werden und die (lokal-)staatlichen Institutionen zum Adressaten haben. Damit aber gehe zugleich eine Erosion sozialer (Klassen)Homogenität zugunsten der Ausbildung sozialstrukturell indifferenter, mehr um „Lebensbereiche“ als um Positionen in der Klassenstruktur organisierter Gruppen einher. Deren konkrete politische Forderungen lösen sich deshalb nicht umstandslos in solchen der Klassenauseinandersetzung auf, sondern können davon losgelöst klassenübergreifende bzw. -indifferente Zielstellungen verfolgen. Wie schon in den Anmerkungen von Engels (1984) zur Wohnungsfrage fehlt es daher auch in den Beobachtungen zum Problem der kollektiven Konsumtion nicht an Skepsis gegenüber politischen Forderungen auf dem Feld der Reproduktion. Anders als die Ausführungen von Engels sind sie aber auch darauf angelegt, die Forderungen und Aktionen der klassenindifferenten, lokalen Bürgerinitiativen an das Ziel der „Abschaffung der Herrschaft des Kapitals“ (Offe, 1972:167) zurückzubinden.

\subsection{Die feminisierte Reproduktionsarbeit und die Reproduktionsarbeitskrise}

Gegenüber der marxistischen Kapitalismusanalyse und der neo-marxistischen Analyse des wohlfahrtsstaatlich abgestützten Spätkapitalismus haben feministische Positionen hervorgehoben, dass der Prozess der Reproduktion der Arbeitskraft (und damit das Reproduktionsniveau) nicht allein auf einer ökonomischen Grundlage - auf dem für zur Verfügung stehenden (Lohn-)Einkommen - fußt oder durch politisch-staatlich organisierte Muster kollektiver Konsumtion determiniert ist, sondern auch und vor allem durch oftmals unentlohnte Haus- und Sorgearbeiten gewährleistet wird. Diese Reproduktionsarbeit umfasst all die Tätigkeiten, die zur Alltagsbewältigung dazu gehören, häufig aber weder als Lohnarbeit organisiert sind noch als Freizeit bezeichnet werden können: Das Putzen und Kochen, die Pflege von Kranken, die Kinderbetreuung und -erziehung, die Organisation von Erholung etc. Solche Tätigkeiten werden regelmäßig von Frauen ausgeübt. Ihnen kam und kommt - auch wenn sie selbst einer Erwerbsarbeit nachgehen - zumeist die Rolle zu, im Produktionsbereich verausgabte Arbeitskraft immer wieder unentlohnt zu reproduzieren.

Mit der Hervorhebung des stark vergeschlechtlichten Charakters der Trennung der Sozialverhältnisse in produktive und reproduktive Bereiche ist es gelungen, die Analyse und Kritik der kapitalistischen Produktionsweise mit der von ungleichen Geschlechterverhältnissen zu verzahnen. Die zentrale Rolle feminisierter Reproduktionsarbeit für die Wiederherstellung der im Produktionsprozess verausgabten Arbeitskraft (und damit für den Akkumulationsprozess des Kapitals) lässt das gesellschaftliche Ungleichheitsverhältnis zwischen den Geschlechtern zumindest in Wechselwirkung, wenn nicht gar als konstitutiv für einen gelingenden Reproduktionsprozess der kapitalistischen Klassengesellschaft insgesamt erscheinen. Und umgekehrt hat der Hinweis auf eine politisch-ökonomische Funktion ungleicher Geschlechterverhältnisse ermöglicht, letztere in Abhängigkeit von Ansprüchen und Erfordernissen der kapitalistischen Klassengesellschaft zu untersuchen (Beer, 1990; Haug, 1999; Haug und Hauser, 1992).

Eine aktuelle, aus dieser Verzahnung gewonnene Situationsdiagnose setzt an der Beobachtung einer Krise der Reproduktionsarbeit an (Bock et al., 1997; Dück, 2014; Winker, 2015; vgl. hier und im Folgenden auch Kemper und Mösgen, 2017:48ff). Demnach ist das traditionelle FamilienernährerModell zur Bewältigung von Reproduktionsaufgaben in den vergangenen Jahrzehnten erheblich unter Druck geraten und steht, so schon Bock et al. (1997), zumindest in Großstadtregionen mit moderner ökonomischer Struktur und den ihnen korrespondierenden Lebensentwürfen als gesellschaftspolitischer Orientierungspunkt gegenwärtig kaum noch zur Verfügung. Doch auch die Orientierung auf den dominanten Alternativentwurf, auf das Zwei-VerdienendeModell, verläuft nicht reibungs- oder problemlos. Das Modell ist zwar der zwangsläufige Orientierungspunkt für Familienhaushalte mit knappen Erwerbseinkommen. Und es ist eine Konsequenz der Forderungen von Frauen nach ihrer Integration in die Erwerbsarbeitsmärkte, die Autonomie und soziale Absicherung versprechen. Das Zwei-VerdienendeModell korrespondiert aber auch mit den gegenwärtigen ökonomischen Akkumulationserfordernissen und den politischökonomischen Absichten zu einer Entlastung der sozialen Sicherungssysteme. Eine gesellschaftliche Durchsetzung dieses Modells allerdings stößt - insbesondere in der 
Variante eines doppelten Vollzeit-Erwerbseinkommens auf Schwierigkeiten. Vor dem Hintergrund einer anhaltend asymmetrisch ausfallenden Aufteilung der Reproduktionsarbeit zwischen den Geschlechtern stellt sich das Modell für Frauen regelmäßig als Doppelbelastung durch sowohl (entlohnte) Erwerbsarbeit wie auch durch (nicht-entlohnte) Haus-und Sorgearbeiten dar.

Dieser Doppelbelastung zu entgehen, so eine der zentralen Beobachtungen zur Krise der Reproduktionsarbeit, war und ist für viele Frauen nur über eine - ihnen zugleich zunehmend unmöglich wie unerwünscht gewordene - eingeschränkte Erwerbstätigkeit oder eben über eine Vermeidung von unentlohnten Reproduktionsaufgaben (etwa durch einen Verzicht auf Kinder und den daran anhängigen Versorgungsund Erziehungsaufgaben) möglich gewesen - was wiederum die Symptomatik der Reproduktionsarbeitskrise eskalieren lässt. Um diese Problematik aufzulösen, haben sich in den letzten Dekaden zwei verschiedene gesellschaftspolitische Strategien entfaltet. Erstens eine Vermarktlichung von Reproduktionsaufgaben: Über die Marktorientierung wird eine Externalisierung von Reproduktionsarbeit an Dritte (zumeist an andere Frauen; Schwiter et al. 2015), ihre Gewährleistung über privatwirtschaftlich organisierte Güterund Dienstleistungsangebote angestrebt. Zweitens eine politische Restrukturierung der Reproduktionsverhältnisse: Über staatliche Interventionen werden ökonomische, rechtliche und zeitliche Ressourcen sowohl für eine Auslagerung von Reproduktionsaufgaben wie für Veränderungen in der vergeschlechtlichten Arbeitsteilung bereitgestellt. Diese Zielstellungen werden in der Bundesrepublik Deutschland seit Mitte der 2000er Jahre vor allem auf drei Wegen verfolgt (vgl. Menke und Klammer, 2017): Über Umstellungen in den familienpolitischen Geldleistungen, Neugestaltungen in den Rechten auf berufliche Aus- bzw. Erziehungszeiten sowie über den Ausbau der Infrastrukturen zur Tagesbetreuung von Kindern (insbesondere unter drei Jahren) wird versucht, den sowohl sozial-, arbeitsmarkt- und gleichstellungspolitisch erwünschten Restrukturierungen der Reproduktionsverhältnisse nachzukommen, die unter dem Leitmotiv einer „Vereinbarkeit von Beruf und Familie“ auf eine Modernisierung der Familienorganisation gerichtet sind.

Einer Vermarktlichung von Reproduktionsaufgaben sind enge sozio-ökonomische Grenzen gesetzt. Ein Rückgriff auf eine Marktversorgung mit Dienstleistungsangeboten für Reproduktionsaufgaben steht nur denjenigen Bevölkerungsteilen zur Verfügung, die über die entsprechenden Mittel dazu verfügen. Eine gesellschaftspolitische Orientierung auf die Kommodifizierung von Reproduktionsaufgaben zugunsten einer Bewältigung der Reproduktionsarbeitskrise droht deshalb sozio-ökonomische Ungleichheiten der Produktionsauf die der Reproduktionssphäre durchschlagen zu lassen und somit eine soziale ,Spaltung innerhalb des Reproduktionsbereichs“ (Siebel, 2012:473) zu evozieren. Die Reproduktionsarbeitskrise wirkt so insbesondere in Großstadtregionen mit einer modernen Dienstleistungsökonomie und der deshalb stark ausdifferenzierten Einkommens- und Sozialstruktur sozial stark selektiv, wird somit insgesamt eher verschärft denn bewältigt.

Demgegenüber versprechen die gegenwärtigen sozialund familienpolitischen Maßnahmen eine allgemeine Entschärfung der Krise der Reproduktionsarbeit (und damit des Reproduktionsprozesses der Arbeitskraft). Die staatlich bereitgestellten materiellen, zeitlichen und infrastrukturellen Ressourcen zugunsten einer Vereinbarkeit von Erwerbsund Reproduktionsarbeit wirken zumindest auf einen ersten Blick auf eine Senkung bzw. Stabilisierung der Reproduktionskosten von Haushalten mit Reproduktionsaufgaben hin. Insbesondere in den urbanen Agglomerationsräumen, in denen ein wachsendes Ungleichgewicht zwischen Einkommens- und Wohnkostenentwicklung das Reproduktionsniveau vieler Haushalte unter Druck gesetzt hat (Kuschinski, 2019), können die Neugestaltung der familienpolitischen Transferzahlungen und eine Intensivierung von infrastrukturellen Angeboten der kollektiven Konsumtion in der frühkindlichen Tagesbetreuung zu einer finanziellen Entlastung von Haushalten mit Reproduktionsaufgaben und damit zu einer Stabilisierung ihres Reproduktionsniveaus beitragen. Auf einen zweiten, analytischen Blick hin wird allerdings deutlich, dass die prinzipielle Programmatik der familienpolitischen Instrumente zur Bewältigung der Reproduktionsarbeitskrise verteilungspolitische Effekte impliziert, die gegebene sozio-ökonomische Ungleichheiten eher verstärken als abschwächen, somit zugleich sozial ungleiche Bearbeitungschancen der Reproduktionsarbeitskrise bedingen (vgl. Kemper und Mösgen, 2017; Menke und Klammer, 2017; Winker, 2015). Zugleich öffnen sich in der Anwendung der Instrumente politische Spielräume auf unterschiedlichen politischen Maßstabsebenen. Zumindest in der quantitativen wie qualitativen Ausgestaltung der Organisation des frühkindlichen Tagesbetreuungsangebots haben, wie ein Vergleich der Stadt- und Landkreise verdeutlicht (Statistisches Bundesamt, 2019), regional unterschiedliche Politiken der Reproduktion stark differierende Muster der kollektiven Konsumtion von Betreuungsmöglichkeiten und somit der Bewältigungsmöglichkeiten der Krise der Reproduktion eröffnet.

Vor diesem Hintergrund untersuchen wir im Folgenden entlang der städtisch-staatlichen Interventionen in die Felder „Wohnen“ und „Kinder betreuen“ im Stadtstaat Hamburg Absichten zu einer urbanen Politik der Reproduktion, die dazu ansetzt, über eine Regulation der Reproduktionsverhältnisse soziale Probleme zu minimieren. Dabei ist sowohl $\mathrm{zu}$ berücksichtigen, wie und für wen ein sozialer Ausgleich angestrebt wird, als auch in den Blick zu nehmen, wie diese Maßnahmen mit den Zwängen einer wachstums- und wettbewerbsorientierten Stadtpolitik vereinbart werden, also eine Strategie der Legitimations- wie Akkumulationssicherung gleichermaßen verfolgt wird. 
3 Urbane Reproduktionsverhältnisse und Stadtpolitik: Wohnen und Kinder betreuen in Hamburg

Die Reproduktionsbereiche „Wohnen“ und „Kinder betreuen" stehen seit mindestens einer Dekade mit im Zentrum der Hamburger Stadtpolitik - zunächst als ein Problem der Alltagsbewältigung, dann als eine von Protestbewegungen skandalisierte gesellschaftliche Zumutung, schließlich ein politisch-administrativ aufgegriffenes und bearbeitetes soziales Problem.

In Hamburg haben zum einen stadtpolitische Debatten um und Proteste gegen Stadtentwicklungsvorhaben, die Gentrifizierung von Stadtvierteln und steigende Mieten große Aufmerksamkeit auf das Feld „Wohnen“ gelenkt. Seit 2009 ist, wie die Entwicklung des Mietspiegels zeigt, der Druck auf den Wohnungsmarkt besonders stark angestiegen (Metzger und Schipper, 2017; Vogelpohl und Buchholz, 2017). Vor diesem Hintergrund hatte die Hamburger Sozialdemokratie auf Wohnungspolitik als das zentrale Wahlkampfthema gesetzt und nicht zuletzt deshalb 2011 die absolute Mehrheit im Hamburger Stadtparlament wiedererlangt. Seither heißt es: „Der Wohnungsbau hat [...] oberste Priorität für den Senat“ (FHH, 2017c:3). Zugleich wird die Bezahlbarkeit von Wohnraum als Politikziel betont (ebd.).

Zum anderen ist in Hamburg der Ausbau der Infrastruktur für eine frühkindliche Betreuung außerhalb der Familie im letzten Jahrzehnt stark vorangetrieben worden und erscheint im Vergleich der Bundesländer bereits weit fortgeschritten. Während anderswo deshalb unter dem Schlagwort „Kitakrise“ nach wie vor der Mangel an Betreuungsplätzen Anlass für politische Proteste gibt und für politischen Handlungsdruck sorgt, sind in Hamburg bereits früh die elterlichen Beiträge für die Betreuung in den Vordergrund gerückt. Frühkindliche Tagesbetreuung über die Einführung einer kostenlosen Grundbetreuung noch attraktiver zu machen und Haushalte mit Kindern von ihren Betreuungskosten zu entlasten, war deshalb ein den Wahlkampf 2011 tragendes Versprechen der SPD in Hamburg und bestimmte das politisch-administrative Handeln auf diesem Gebiet in den Folgejahren.

\subsection{Neubau und Sozialwohnungen: Stadtpolitische Antworten auf die Wohnungskrise}

Für Hamburg wird sowohl hinsichtlich der Wohnkosten als auch des Wohnungsbestandes von einer Wohnungsnot bzw. Wohnungskrise gesprochen (Metzger und Schipper, 2017; Mieter helfen Mietern Hamburg et al., 2018). In Hamburg sind $75 \%$ der Wohnungen Mietwohnungen (Statistisches Landesamt Bremen et al., 2016:52). Als ein Indikator für die Belastung mit Wohnkosten im Mietwohnungssegment gilt die Mietbelastungsquote. Der Schwellenwert für eine annehmbare Mietbelastung ist demnach ein Anteil von $30 \%$ des Haushaltsnettoeinkommens. Dieser Wert wird auch vom Hamburger Senat als das Maximum für eine Bewertung von Wohnraum als „bezahlbar“ angesehen (FHH, 2017b:4). Was darüber hinaus geht, ist insbesondere für Haushalte mit geringem Einkommen problematisch, weil das übrig bleibende Einkommen dann sehr niedrig ausfällt (Lebuhn et al., 2017). Die durchschnittliche Mietbelastungsquote durch die Nettokaltmiete beträgt in Hamburg 28,3\%; hinzu kommen Heiz- und Energiekosten, die in der Regel nochmal etwa $5 \%$ bis $6 \%$ ausmachen. Damit zählt Hamburg bereits zu den Top 10 unter den deutschen Städten, was die Mietbelastung betrifft, und die Stadt führt die Liste gemeinsam mit Düsseldorf, Neuss und Bonn an, in denen arme Haushalte für die Kaltmiete $46 \%$ des Einkommens aufbringen müssen (Lebuhn et al., 2017:73). In Hamburg sind die Wohnkosten für etwa ein Drittel der Hamburger*innen (bzw. $45 \%$ der Mieter*innen) als kritisch einzustufen. ${ }^{1}$

Hinsichtlich des Wohnungsbestandes ist ebenfalls eine kritische Situation festzustellen. Gegenwärtige Prognosen gehen von einem Bevölkerungszuwachs von etwa jährlich 10000 neuen Einwohner*innen bis 2030 aus. Das entspricht einem Zuwachs von ca. 5000 neuen Haushalten jedes Jahr (FHH, 2017a:14). Im Kontext der steigenden Konkurrenz um Wohnraum sind nicht nur arme Haushalte berechtigt, eine geförderte Wohnung (Sozialwohnung) zu beziehen, sondern auch solche mit niedrigem Einkommen. Aktuell sind dies $41 \%$ der Hamburger Haushalte; wird der 2. Förderweg, der sich an Haushalte mit einem etwas höherem Einkommen richtet, hinzugerechnet, haben $55 \%$ der Haushalte einen Anspruch auf eine Sozialwohnung. Diesen anspruchsberechtigten 365000 Haushalten stehen aber nur knapp 80000 Sozialwohnungen gegenüber (alle Zahlen aus: Bürgerschaft der FHH, 2017). Gegenwärtig bietet die Stadt Hamburg also nur $20 \%$ der zugangsberechtigten Haushalte eine Sozialwohnung an. Darüber hinaus gibt es in Hamburg ca. 14000 Haushalte, die als Wohnungsnotfälle gelten und auf dem Wohnungsmarkt unversorgt bleiben (Mieter helfen Mietern Hamburg et al., 2018).

Diese Zahlen zeigen, dass die Wohnungsfrage eine klassenübergreifende ist - sie betrifft nicht nur Personen unter der Armutsgrenze, sondern reicht weit in die Mittelschicht hinein. Die konkrete sozialdemokratische Antwort auf diese Situation lautet seit 2011: (a) Forcierung des Wohnungsneubaus, verbunden mit (b) einem „Drittelmix“, der neben einem Drittel Eigentumswohnungen und einem Drittel frei vermieteter Wohnungen auch ein Drittel geförderte Wohnungen gewährleisten soll. Mietenpolitik im Bereich der Bestandsmieten wird nur wenig betrieben, lediglich die relativ schwache Mietpreisbremse

\footnotetext{
${ }^{1}$ https://www.haspa.de/content/dam/myif/haspa/work/ dokumente/pdf/haspa/presse/haspa-trendbarometer/ 2018-trendbarometer-wohnen.pdf?n=true, letzter Zugriff: 9. März 2018.
} 
und Soziale Erhaltungsverordnungen (vgl. Vogelpohl und Buchholz, 2017). Hinsichtlich des Wohnungsbestandes war es dabei zunächst das Ziel, jährlich 6000 neue Wohnungen zu genehmigen. Ab 2017 wurde das Ziel auf $10000^{2}$ Wohnungen erhöht (FHH, 2017c). Hinsichtlich der Wohnkosten erwartet der Senat von dem neuen Angebot an Wohnungen und den darin entstehenden Sozialwohnungen eine dämpfende Wirkung auf den Anstieg der Mietpreise (FHH, 2017b:23). Mit dem anvisierten Drittelmix, so das Versprechen (FHH, 2017b), könne allen Ansprüchen von aktuellen wie künftigen Bewohner*innen auf preisgünstigen Wohnraum Rechnung getragen werden. Trotzdem sinkt der Anteil an Sozialwohnungen kontinuierlich. Denn da die Mietpreisbindung stets befristet ist, werden mehr Sozialwohnungen zu frei vermieteten Wohnungen, als neue Wohnungen mit Bindungen belegt werden: Im Jahr 2016 waren noch $8,4 \%$ aller Wohnungen Sozialwohnungen; bis zum Jahr 2022 fallen davon ein Viertel, nämlich jährlich ca. 3800 Wohnungen, aus der Bindung heraus (Statistikamt Nord, 2018).

Im Spiegel des aktuellen Status quo und daraus ableitbaren Ansprüchen an preisgünstige Mietverhältnisse zeigt sich eine soziale Schieflage, die in der gegenwärtigen Wohnungspolitik in Hamburg angelegt ist: Gegenwärtig leben nicht ein Drittel, sondern nur ein Viertel der Hamburger Wohnungen in selbstbewohntem Eigentum. Und der Bedarf an Sozialwohnungen liegt nicht nur bei einem Drittel, sondern, wie bereits erwähnt, bei gut $40 \%$ (1. Förderweg) bzw. $55 \%$ (inkl. 2. Förderweg). Der jährliche Zuwachs an Sozialwohnungen reicht ganz offensichtlich nicht aus, um die jährlich aus der Sozialbindung fallenden Wohnungen $\mathrm{zu}$ ersetzen. Dies ist besonders problematisch, weil auch der Anteil an Wohnungen im 2. Förderweg sukzessive gesteigert werden soll, von aktuell nur 2,5\% auf über $25 \%$ (Bürgerschaft der FHH, 2017). Deswegen werden bis 2030 im 1. Förderweg nur noch 60000 Wohnungen existieren.

Die sozialdemokratische Wohnungspolitik der letzten Jahre wirkt deshalb ambivalent: Einerseits wird tatsächlich ein intensiverer Wohnungsbau betrieben als in der Periode christdemokratisch geführter Stadtpolitik zwischen 2001 und 2011. In diesen Jahren wurden jährlich ca. 3000 Wohnungen gebaut; seit 2011 ist der Wohnungsneubau auf knapp 7000 Wohnungen jährlich gesteigert worden. Zudem sind die Förderzusagen im sozialen Wohnungsbau von ca. $1500 \mathrm{im}$ Jahr 2010 auf über 3300 im Jahr 2016 erhöht worden; die Fördersumme im Wohnungsbau wurde von EUR 120 Mio. im Jahr 2010 sukzessive auf EUR 250 Mio. im Jahr 2018 gesteigert $^{3}$. Andererseits repräsentiert die

\footnotetext{
${ }^{2}$ Mit der Ausweitung des Plans auf 10000 neue Wohnungen wurde stillschweigend das Ziel von 3000 geförderten Wohnungen eingeführt (FHH, 2017c), was nicht mehr einem Drittel entspricht.

${ }^{3}$ Zahlen aus den IFB-Jahresberichten unter https://www. ifbhh.de/downloads?tab=allgemeine-informationen, letzter Zugriff: 6. April 2020. Die Summen spiegeln jegliche Förderung im
}

sozialdemokratische Wohnungspolitik eine grundlegend unternehmerische Stadtpolitik. Mit dem Drittelmix macht die Politik vorrangig jenen Einkommensgruppen ein Angebot, die relativ hohe Mieten zahlen bzw. sich Wohneigentum in der Stadt schaffen können: zwei Drittel des Mix richten sich an Haushalte ohne irgendeinen Förderbedarf. Große Neubaugebiete richten sich darüber hinaus vor allem an Familien - Familien hier im Sinne von Haushalten mit zwei Erwachsenen und Kindern. Diese Haushalte stellen aber derzeit lediglich $13,3 \%$ der Hamburger Haushalte dar. Zugleich ist jeder vierte Familienhaushalt ein Alleinerziehendenhaushalt (Statistikamt Nord, 2019:42). Mittelschichtsfamilien, die sich das Wohnen im Neubau leisten können, sind die Schicht, die für den städtischen Haushalt besonders attraktiv ist, weil sie weniger kostet als Steuern einzahlt und zahlenmäßig so stark ist, dass sich eine darauf zugeschnittene Politik lohnt (im Gegensatz zu Haushalten mit hohem und sehr hohem Einkommen, vgl. Herrmann, 2010). Die Wohnungskrise selbst bleibt mit den aktuellen Ansätzen vor allem auf Ebene der Wohnkosten bestehen und verschärft sich hier sogar mit den teuren Neubauten, die überproportional in den Mietspiegel eingehen.

Diese städtische Sozialpolitik im Bereich Wohnen, mit der sich die SPD wieder in die Regierung manövrieren konnte, dient erstens der Abfederung sozialer Ungleichheiten. Überhaupt wieder stärker Sozialwohnungen zu fördern, vermeidet weitere Polarisierungen. Besonders ersichtlich ist dies im seit vielen Jahren gleichbleibenden Sozialmonitoring der Stadt, wo sich die Behörde für Stadtentwicklung stets positiv über fehlende Dynamik im Sinne einer Segregation äußert. Fast wortgleich wird der Bericht jedes Jahr kommentiert: „Erneut zeigt der aktuelle Sozialmonitoring-Bericht eine sehr stabile sozialräumliche Entwicklung für Hamburg auf. Erfreulich ist, dass nach wie vor keine zunehmenden Polarisierungstendenzen in Hamburg erkennbar sind“ (BSW, 2019:5). Zweitens ist die sozial erscheinende Wohnpolitik eine Förderung der Produktions- und Reproduktionswirtschaft par excellence: private Projektentwicklungsagenturen werden $\mathrm{zu}$ vermehrten Bauen angeregt, wodurch vor allem Neubauprojekte entstehen, die die gut bis sehr gut verdienende Mittelschicht adressiert (dem Drittelmix zum Trotz). Für diese Mittelschicht wird die Gleichzeitigkeit von Erwerbs- und Reproduktionsarbeit durch infrastrukturell gut angeschlossene Neubaugebiete ermöglicht, während ökonomisch schlechter gestellte Familien und Alleinerziehende durch diese Politik kaum direkte Unterstützung erfahren (dazu Kuschinski und Reichle, 2020). Die Abfederung durch Sozialwohnungen verschafft dieser Politik Legitimation, während die Neubauförderung Akkumulation fördert und unbezahlte Reproduktionsarbeit sichert.

Wohnungsbau wider und schließen neben sozialem Wohnungsbau auch energetische oder barrierefreie Modernisierungen ein. 
Dass diese Wohnungspolitik Teil einer die Reproduktionspolitik ist, die ein Doppelverdienst-Haushaltsmodell mit Teilzeit-Anteilen anstrebt, wird in der Zusammenschau mit der lokalen Kita-Politik deutlich.

\subsection{Ausbau und Kostenbefreiung: Stadtpolitische Antworten auf die Kitakrise}

Im Jahr 2007 wurden bundesweit 15,5\% aller Kinder unter drei Jahren tagsüber in einer Kindertagesstätte oder von Tageseltern betreut, in Hamburg waren es damals bereits $22 \%$ (Statistisches Bundesamt, 2008:28, 36). Etwas mehr als zehn Jahre später, im Jahr 2019, liegt die Betreuungsquote im Bundesdurchschnitt bei $34,3 \%$. In Hamburg werden inzwischen $46,6 \%$ aller Kinder unter drei Jahren außerfamiliär betreut ${ }^{4}$.

Die in Hamburg inzwischen erreichte, vergleichsweise hohe Betreuungsquote von Kindern unter drei Jahren reflektiert eine städtisch-staatliche Angebotspolitik familienpolitischer Infrastruktur, die insbesondere seit dem Wahlerfolg der Hamburger SPD von 2011 nicht allein den quantitativen Ausbau der Möglichkeiten einer Tagesbetreuung für Kinder unter drei Jahren forciert, sondern auch auf qualitative Anreize zu deren Wahrnehmung setzt. Hauptanreizmomente des Angebots eines kollektiven Konsums frühkindlicher Tagesbetreuung in Hamburg sind (siehe etwa Boll und Lagemann, 2017):

1. Ein Gutschein-System, das Kindern bzw. ihren Eltern prinzipiell die freie Wahl zwischen verschiedenen Angeboten der frühkindlichen Tagesbetreuung ermöglicht (seit August 2003).

2. Eine zeitliche Ausdehnung des Rechtsanspruchs auf frühkindliche Tagesbetreuung auf fünf Stunden täglich (seit August 2012 für Kinder ab dem zweiten, seit August 2013 für Kinder ab dem ersten vollendeten Lebensjahr).

3. Eine über ein allgemeines, bedarfsunabhängiges und gebührenfreies „Kita-Basis-Angebot“ besorgte finanzielle Entlastung der Familien bei Wahrnehmung des Rechtsanspruchs (seit August 2014).

Eine fünfstündige Betreuung täglich bleibt demnach kostenfrei. Für einen darüber hinausgehenden, durch Erwerbsarbeit, Arbeitssuche oder Ausbildung begründbaren Betreuungsbedarf werden weiterhin Elternbeiträge erhoben. Diese fallen je nach dem Haushaltsnettoeinkommen, der Familiengröße und dem Betreuungsumfang gestaffelt aus, allerdings eingerahmt durch Mindest- und Höchstbeiträge.

Während das Gutschein-System anscheinend noch die marktorientierte Grundhaltung der christdemokratisch geführten Stadtpolitik der 2000er Jahren widerspiegelt, sind

\footnotetext{
${ }^{4}$ https://www.destatis.de/DE/Themen/Gesellschaft-Umwelt/ Soziales/Kindertagesbetreuung/Tabellen/betreuungsquote-2018. html, letzter Zugriff: 3. April 2020.
}

die Ausdehnung des Rechtsanspruchs und die finanzielle Entlastung Ausdruck der Politik der sozialdemokratisch geführten Stadtregierung seit 2011. Sie zielen nach Senatsmeinung darauf $a b$, die Zugangshürden zur frühkindlichen Tagesbetreuung abzubauen, die Lebenshaltungskosten von Haushalten mit Kindern erheblich zu reduzieren und somit „Hamburg als Wohnort für junge Familien noch attraktiver" ${ }^{\text {"5 }}$ zu machen.

Die propagierte Zielgruppe ,junge Familien“ lässt sich mit Hilfe einer Rekonstruktion des Ausmaßes der finanziellen Entlastung für verschiedene Einkommensgruppen sozialanalytisch näher beleuchten. Tabelle 1 verdeutlicht am Modell einer dreiköpfigen Familie (zwei Erwachsene, ein Kind) mit einem Betreuungsbedarf von zehn Stunden drei unterschiedliche Effekte der allgemeinen Entlastung für unterschiedliche Einkommensgruppen. Demnach fällt (a) die absolute Kostenreduktion für Familien mit höheren monatlichen Haushaltsnettoeinkommen höher aus als für solche mit niedrigeren. ${ }^{6}$ Von (b) der relativen Kostenreduktion dagegen profitieren insbesondere solche Familien, deren Einkommen unterhalb des Hamburger Einkommensmedians (für eine dreiköpfige Familie) von knapp EUR $3000^{7}$ liegen. Für einen Großteil dieser Haushalte haben sich die Kosten um $60 \%$ und mehr reduziert. Familien mit einem Haushaltseinkommen über dem Median dagegen sehen sich um knapp 50\% entlastet. Ein Blick auf (c) die Veränderung der Betreuungsbelastungsquote ${ }^{8}$ wiederum zeigt eine relative Begünstigung vor allem von Familien mit mittleren, um den Median liegenden Einkommen zwischen EUR 2000 und 4500. Für sie reduziert sich der Anteil der Aufwendungen für die Kindertagesbetreuung am Haushaltsnettoeinkommen um 4 Prozentpunkte und mehr.

Anhand der Betreuungsbelastungsquote lässt sich darüber hinaus auch die allgemeine verteilungspolitische Rationalität der Gebührenstruktur des Elternbeitrags zur Kinderbetreuung rekonstruieren. Der Elternbeitrag ist vor wie nach der Einführung der beitragsfreien Grundbetreuung als ein

\footnotetext{
${ }^{5}$ So etwa in Bürgerschaft der FHH (2014:2); siehe auch http: //www.hamburg.de/elterninformationen/116832/elternbeitraege/, letzter Zugriff: 22. Mai 2018.

${ }^{6}$ Die zusätzlichen Gelder durch das „Gute-Kita-Gesetz“ des BMFSFJ setzt Hamburg seit August 2019 für eine vollständige Gebührenbefreiung für Eltern, die staatliche Transferleistungen erhalten, ein.

${ }^{7}$ Zur Einkommenssituation von Familien in Hamburg liegen offenbar keine regelmäßig erfassten und systematisch aufbereiteten Daten vor (BASFI, 2017:38). Entlang der Angaben der amtlichen Sozialberichterstattung zur Armutsgefährdungsschwelle (Statistisches Bundesamt, 2020) allerdings lässt sich für eine dreiköpfige Familie (zwei Haushaltmitglieder über und ein Haushaltsmitglied unter 14 Jahren in 2014) ein Einkommensmedian von EUR 2892 rekonstruieren.

${ }^{8}$ In Analogie zur Mietbelastungsquote bezeichnen wir als Betreuungsbelastungsquote den Anteil am Haushaltsnettoeinkommen, der für die frühkindliche Tagesbetreuung aufgebracht werden muss.
} 
Tabelle 1. Elternbeiträge für die frühkindliche Tagesbetreuung, finanzielle Entlastung und Betreuungsbelastungsquote (Modellierung für einen Haushalt mit drei Personen, davon ein Kind, täglicher Betreuungsbedarf bis zu zehn Stunden).

\begin{tabular}{lrr|rr|rrr}
\hline $\begin{array}{l}\text { Monatl. Netto- } \\
\text { Einkommen }\end{array}$ & \multicolumn{2}{c|}{$\begin{array}{c}\text { Beitrag in } \\
\text { Euro }\end{array}$} & \multicolumn{2}{c|}{$\begin{array}{c}\text { Finanzielle } \\
\text { Entlastung }\end{array}$} & \multicolumn{3}{c}{$\begin{array}{c}\text { Betreuungsbelastungs- } \\
\text { quote }\end{array}$} \\
\cline { 2 - 8 } in Euro & 2013 & 2014 & in Euro & in Prozent & 2013 & 2014 & Differenz \\
\hline 1000 & 43 & 16 & 27 & 62,8 & 4,3 & 1,6 & 2,7 \\
1500 & 60 & 23 & 37 & 61,7 & 4,0 & 1,5 & 2,5 \\
2000 & 144 & 55 & 89 & 61,8 & 7,2 & 2,8 & 4,5 \\
2500 & 262 & 99 & 163 & 62,2 & 10,5 & 4,0 & 6,5 \\
3000 & 396 & 204 & 192 & 48,5 & 13,2 & 6,8 & 6,4 \\
3500 & 396 & 204 & 192 & 48,5 & 11,3 & 5,8 & 5,5 \\
4000 & 396 & 204 & 192 & 48,5 & 9,9 & 5,1 & 4,8 \\
4500 & 396 & 204 & 192 & 48,5 & 8,8 & 4,5 & 4,3 \\
5000 & 396 & 204 & 192 & 48,5 & 7,9 & 4,1 & 3,8 \\
\hline
\end{tabular}

Quellen: BASFI (2013, 2014). Eigene Berechnung und Darstellung.

Progressionsmodell konzipiert, aber eingerahmt durch einen Eingangs- und einen Höchstbeitrag. Letzterer ist, wie Abb. 1 verdeutlicht, offenbar am Median der Einkommen orientiert. Für Familien mit einem Haushaltseinkommen unterhalb des Median von knapp EUR 3000 steigt demnach die Betreuungsbelastungsquote zusammen mit einem steigenden Haushaltseinkommen an. Familien, deren Haushaltseinkommen über dem Median von knapp EUR 3000 liegen, müssen dagegen wegen des konstant bleibenden Höchstbeitrags mit wachsendem Einkommen einen zunehmend geringeren Anteil ihres Haushalteinkommens für die Kinderbetreuung aufwenden. An dieser, tendenziell die über dem Median liegenden Haushaltseinkommen privilegierenden Gebührenstruktur ist auch mit der Einführung des beitragsfreien „Basis-Angebots“ nicht gerührt worden.

Die Einführung des beitragsfreien „Basis-Angebots“ weist drei Momente einer städtischen Reproduktionspolitik auf: Erstens ist die Veränderung der Betreuungsgebühren ein klassenübergreifendes Projekt; es reduziert die Aufwendungen für alle Einkommensgruppen und nicht im Besonderen die der Einkommensschwachen. Im Gegenteil. Die Gebührenstruktur privilegiert verstärkt die über dem Median liegenden Haushaltseinkommen, weil vor allem Familien der unteren und oberen Mitte entlastet worden sind. Die Senkung der Lebenshaltungskosten der privaten Haushalte mit Kindern ist zweitens eine Stärkung des kollektiven Konsums: die tendenzielle Vergesellschaftung der Betreuungskosten durch den lokalen Staat macht sich in Mehrausgaben im städtischen Haushalt geltend. Nach Angaben der Hamburger Behörde für Schule und Berufsbildung ist das „Zuschussvolumen für die Betreuungsangebote im Krippen- und Elementarbereich“ in den letzten Jahren stark angestiegen, ,von rund 275 Millionen Euro im Jahr 2005 auf rund 719 Millionen Euro im Jahr 2015“ (BSB, 2017:47). Die noch geleisteten Elternbeiträge dagegen tragen nur noch $7 \%$ der auf dem Gebiet der Kinderbetreuung anfallenden Kosten

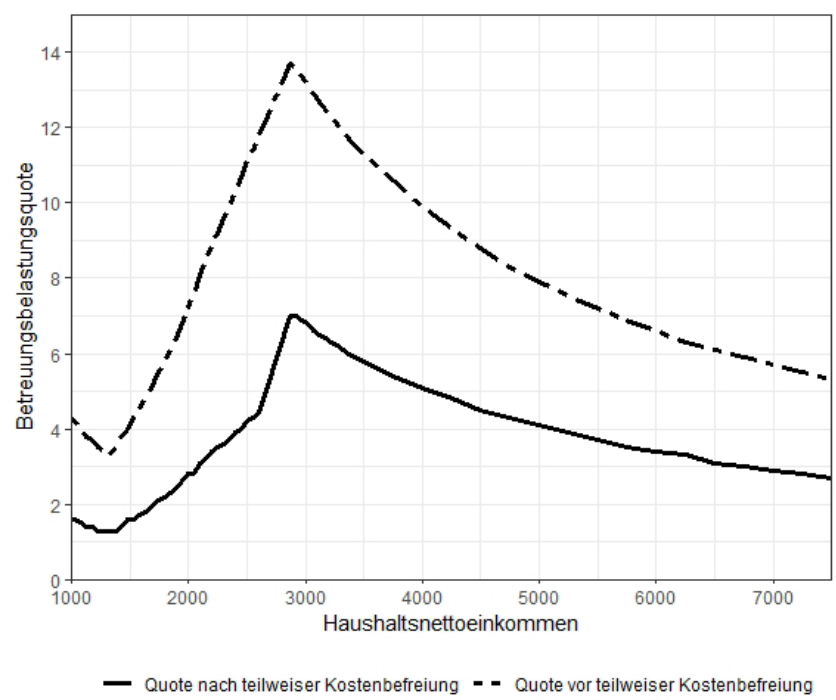

Abb. 1. Betreuungsbelastungsquote vor und nach der Einführung eines beitragsfreien Grundangebots für die frühkindliche Tagesbetreuung (Modellierung für einen Haushalt mit drei Personen, davon ein Kind, täglicher Betreuungsbedarf bis zu zehn Stunden). Quellen: BASFI (2013, 2014). Eigene Berechnung und Darstellung.

(vgl. BSB, 2017). Und drittens spricht das Basisangebot einer fünfständigen Betreuung für die Vorstellung einer Familie, in der mindestens eine Person nur in Teilzeit arbeitet, um ebenfalls unbezahlte Reproduktionsarbeit in Teilzeit leisten zu können.

\section{Die sozial-unternehmerische Stadt: Urbane Sozialpolitik als Reproduktions- und Standortpolitik}

Die neuen Sozialpolitiken im Bereich des Wohnens und der Kinderbetreuung setzen das wettbewerbsorientierte 
Akkumulationsmodell der unternehmerischen Stadt nicht aus, sondern treiben es weiter. Die Sozialpolitik adressiert soziale Ungleichheiten, geht jedoch nicht Ursachen, sondern Symptome einer ungleichheitsproduzierenden Standortpolitik an. Die städtische Politik in Hamburg zielte in den letzten Jahren darauf, auf dem Gebiet der Reproduktion den allgemeinen Druck auf die Lebensverhältnisse zu minimieren, den die explizite stadtpolitische und -planerische Wettbewerbsorientierung der Jahre zuvor evoziert hatte. Das gelingt insbesondere über Neujustierungen der städtischen Wohnungs- und Familienpolitik. Unsere Analyse, wie und für wen die aktuellen Formen städtischer Reproduktionspolitik ausgestaltet werden, macht zusammenfassend drei Bezüge dieser Reproduktionspolitik zur unternehmerischen Stadt deutlich:

1. Entschärfung, klassenübergreifend. Mit einem verstärkten öffentlichen Wohnungsbau und einer tendenziellen Vergesellschaftung der Kosten für die frühkindliche Betreuung werden einige Haushalte von einigen laufenden Kosten entlastet. Die gegenwärtigen Maßnahmen der Wohnungs- und Kinderbetreuungspolitik sind allerdings nicht vorrangig für jene organisiert, die die drängendsten Probleme in einer marktförmig organisierten Reproduktionssphäre haben. Zwar kommen die gesteigerten städtischen Investitionen in den sozialen Wohnungsbau (mehr als eine Verdoppelung seit 2010) und in die Kindertagesbetreuung (fast eine Verdreifachung seit 2005) auch Haushalten mit geringem Einkommen zugute. Das nimmt zudem die Spitze aus einer sich verschärfenden sozialen Ungleichheit. Doch die zentrale Tendenz der beschriebenen Neujustierungen liegt in der Orientierung auf die Lebensbedingungen und -wünsche der mittleren und oberen Einkommensklassen. Das wird etwa am wohnungspolitischen Postulat des „Drittelmix“, der an der gegenwärtigen Sozialstruktur der Stadt vorbei zielt, ebenso deutlich wie an der Beitragsbemessungsgrenze der Elternbeiträge für die frühkindliche Betreuung, die prinzipiell mittlere bis höhere Einkommensklassen privilegiert. Sie werden finanziell entlastet und ihre oftmals artikulierten Sehnsüchte nach einer Politik der sozialen Mischung und des sozialen Ausgleichs (vgl. dazu Frank und Weck, 2018) bedient.

2. Stabilisierung, akkumulationssichernd. Die klassenprivilegierenden Züge der stadtpolitischen Neujustierungen auf den Feldern ,Wohnen“ und „Kinder betreuen" können direkt auf das Ziel bezogen werden, über eine Steigerung der als „Lebensqualität“ vermarkteten Reproduktionsbedingungen für Haushalte der mittleren Einkommensklassen einen Standortvorteil in der Konkurrenz der Städte und Gemeinden um steuerkräftige Haushalte auszubauen. Gekoppelt an die klassenübergreifenden Entlastungen für alle wird das regionale Lohnniveau stabilisiert, so das Prinzip. Ein
Umschlagen der gestiegenen Lebenshaltungskosten in die Forderung nach einer höheren Entlohnung soll blockiert und so die Kapitalverwertungsbedingungen des Standorts Hamburg zumindest auf dem etablierten Niveau gehalten werden. Darin wird auch die Kontur einer lokalen Fachkräftestrategie erkennbar, die eben nicht allein bei den ,neuen“ Mittelschichten und bei kapital- und wissensintensiven Dienstleistungen ansetzt, sondern sich auch auf die Lebensbedingungen der Arbeiter- und Angestelltenmilieus erstreckt, sich also um den Status quo der städtischen Reproduktionsverhältnisse insgesamt sorgt.

3. Absicherung, vergeschlechtlicht. Der unbezahlte Bereich der Reproduktionsbereich wird nicht direkt, aber indirekt in der sozial-unternehmerischen Stadt gesteuert. Die ,Vereinbarkeit von Beruf und Familie“ ist also nicht nur ein individuell ausgehandeltes Problem, sondern auch ein gesellschaftlich verhandeltes. Die Förderung von Wohnen in gut angeschlossenen Neubaugebieten unterstützt die Erwerbsbeteiligung beider Eltern, während die 5-Stunden-Basisbetreuung in Kitas dieses Doppelverdienst-Modell als Modell mit partieller Teilzeit konkretisiert. Befördert wird also ein FamilienHaushaltsmodell, in dem mindestens eine Person in Teilzeit erwerbstätig ist und in der übrig bleibenden Teilzeit unentlohnte Reproduktionsarbeitet leistet. In Teilzeit wiederum arbeiten viel mehr Frauen (im Jahr 2018 in Deutschland und Österreich: 46\%, in der Schweiz: 60\%) als Männer (Deutschland und Österreich: $10 \%$, Schweiz: $17 \%$; Quelle: eurostat). Das Doppelverdienst-Modell mit Teilzeit-Anteilen basiert also auf ungleichen Geschlechterverhältnissen; diese werden durch die Politik der sozial-unternehmerischen Stadt abgesichert.

Diese drei Aspekte möchten wir als Kontur der sozialunternehmerischen Stadt verstanden wissen, die noch weiter ausdifferenziert werden kann und muss. Grundsätzlich aber lässt sich erkennen: Mit der Politik einer sozialunternehmerischen Stadt ist keine Aussicht auf eine Verringerung sozialer Ungleichheiten verbunden; vielmehr lenkt sie von diesen weg - und bleibt deswegen auch nicht unhinterfragt:

Erstens gelingt es mit dem bisherigen Ausmaß an Interventionen städtischer Reproduktionspolitik offensichtlich nicht, die stadtpolitischen Auseinandersetzungen stillzustellen. Das Konzept des „Drittelmix“ und die Forcierung des Wohnungsneubaus sind längst konfrontiert mit Forderungen nach einer Reform des Mietspiegels, einer ausschließlichen Entwicklung städtischer Flächen durch die städtische Wohnungsbaugesellschaft SAGA/GWG zu günstigen Preisen oder nach längeren Bindungen im sozialen Wohnungsbau (s. Vorschläge in Mieter helfen Mietern Hamburg et al., 2018). Dem quantitativen Ausbau wie der finanziellen Attraktivität des Hamburger Kindertagesbetreuungsangebots wiederum 
stehen elterliche Klagen über die fragliche Qualität der Betreuung sowie zu den Forderungen der Beschäftigten nach verbesserten Arbeitsbedingungen gegenüber (vgl. Volksinitiative „Mehr Hände für Hamburger Kitas“"9). Wie und für wen urbane Reproduktionspolitiken auf den Feldern „Wohnen“ und „Kinder betreuen“ gemacht wird, steht damit weiterhin zur stadtpolitischen Diskussion.

In dieser Dynamik spiegelt sich, zweitens, ein grundsätzlich widersprüchlicher und tendenziell kollisionsträchtiger Problemzusammenhang wider. Die Implementation unternehmerischer Stadtpolitik über den Reproduktionsbereich der Stadtgesellschaft forciert die relative Stärke der Stadt Hamburg in der Metropolenkonkurrenz. Die so gewonnenen Ressourcen wiederum ermöglichen den finanziellen Spielraum für den Versuch, die Folgeprobleme abzumildern, die die wachstums- und wettbewerbsorientierte Stadtpolitik selbst hervorruft. Der Versuch, die Reproduktionskrise auf städtischer Ebene zu lösen, wird so finanziell von dem Wachstum abhängig gemacht, das als Ursache für die Reproduktionsprobleme zu gelten hat - bis zur nächsten Wirtschaftskrise. Die Corona-Pandemie ist bereits die nächste Krise, die dem Zusammenspiel von Wachstumspolitik und ausgleichender Sozialpolitik die Grundlage entziehen könnte. Es wird zu zeigen sein, ob in dem nun anstehenden Austarieren die unternehmerische oder die soziale Politik an Gewicht gewinnt.

Datenverfügbarkeit. Für diesen Beitrag wurde keine eigenständige Datenerhebung durchgeführt. Die in Abschnitt 3 des Beitrags herangezogenen Statistiken und Beitragsgrößen sind über die benannten Quellen öffentlich zugänglich.

Autorenmitwirkung. Der Beitrag wurde von den Autor*innen gemeinsam und zu gleichen Teilen verfasst.

Interessenkonflikt. Die Autoren erklären, dass kein Interessenkonflikt besteht.

Danksagung. Wir möchten den Herausgebern des Themenheftes, Simon Runkel und Jonathan Everts, für die Einladung zum Schreiben dieses Beitrags und die hilfreiche Kommentierung einer frühen Manuskriptversion danken. Zudem danken wir den drei anonymen Gutachter*innen für ihre sehr ausführliche und hilfreiche Auseinandersetzung mit dem Manuskript.

Begutachtung. This paper was edited by Benedikt Korf and reviewed by three anonymous referees.

\section{Literatur}

Althusser, L.: Über die Reproduktion. Ideologie und ideologische Staatsapparate, 2. Halbband, VSA, Hamburg, Deutschland, 2012.

BASFI (Behörde für Arbeit, Soziales, Familie und Integration): Elternbeiträge für Kindertageseinrichtungen und Kindertagespflege, gültig ab August 2013, Hamburg, Deutschland, 2013.

BASFI (Behörde für Arbeit, Soziales, Familie und Integration): Elternbeiträge für Kindertageseinrichtungen und Kindertagespflege, gültig ab August 2014, Hamburg, Deutschland, 2014.

BASFI (Behörde für Arbeit, Soziales, Familie und Integration, Hrsg.): Familien in Hamburg: Lebenslagenbericht, Hamburg, Deutschland, 2017.

Beer, U.: Geschlecht, Struktur, Geschichte - soziale Konstituierung des Geschlechterverhältnisses, Campus, Frankfurt am Main, Deutschland, 1990.

Bock, S., Heeg, S., und Rodenstein, M.: Reproduktionsarbeitskrise und Stadtstruktur. Zur Entwicklung von Agglomerationsräumen aus feministischer Sicht, in: Durch die Wand! Feministische Konzepte zur Raumentwicklung, Herausgeber: Becker, R. und Bauhardt, C., Centaurus, Pfaffenweiler, Deutschland, 33-52, 1997.

Boll, C. und Lagemann, A.: Elterliche Erwerbstätigkeit und Kinderbetreuung in Hamburg: Entwicklung und Zusammenhänge im Kontext knapper werdender Fachkräfte, Hamburgisches WeltWirtschaftsInstitut (HWWI), HWWI Policy Paper, 103, Hamburg, Deutschland, 2017.

BSB (Behörde für Schule und Berufsbildung, Hrsg.): Bildungsbericht Hamburg 2017, HANSE - Hamburger Schriften zur Qualität im Bildungswesen, 16, Waxmann, Münster, Deutschland, New York, USA, 2017.

BSW (Behörde für Stadtentwicklung und Wohnen Hamburg, Hrsg.): Sozialmonitoring Integrierte Stadtteilentwicklung - Bericht 2019, Hamburg, Deutschland, 2019.

Bürgerschaft der FHH (Bürgerschaft der Freien und Hansestadt Hamburg): Schriftliche Kleine Anfrage des Abgeordneten Christoph de Vries (CDU) vom 08.09.14 und Antwort des Senats, Betr.: Landesrahmenvertrag ,Kinderbetreuung in Tageseinrichtungen" - wie ist der Stand der Verhandlungen?; Drucksache 20/12954, 2014.

Bürgerschaft der FHH (Bürgerschaft der Freien und Hansestadt Hamburg): Schriftliche Kleine Anfrage der Abgeordneten Heike Sudmann (DIE LINKE) vom 19.01.17 und Antwort des Senats, Betr.: Verhältnis von Sozialwohnungen und anspruchsberechtigten Haushalten in Hamburg; Drucksache 21/7630, 2017.

Castells, M.: The Urban Question: A Marxist Approach, Edward Arnold, London, UK, 1977.

Dück, J.: Krise und Geschlecht - Überlegungen zu einem feministisch-materialistischem Krisenverständnis, Prokla, 44, 53-70, 2014.

Engels, F.: Zur Wohnungsfrage, in: Marx-Engels-Gesamtausgabe, Band I.24, Dietz, Berlin, Deutschland, 7-81, 1984.

FHH (Freie und Hansestadt Hamburg, Hrsg.): Eine Stadt für Alle: Wie wollen wir wohnen? Wo können wir bauen?, Hamburg, Deutschland, 2017a.

\footnotetext{
${ }^{9}$ https://www.kitanetzwerk-hamburg.de/die-volksinitiative. html, letzter Zugriff: 20. April 2018.
} 
FHH (Freie und Hansestadt Hamburg, Hrsg.): Wohnen in Hamburg 2030. Eine Stadt für Alle - Strategien für bezahlbares Wohnen, Hamburg, Deutschland, 2017b.

FHH (Freie und Hansestadt Hamburg, Hrsg.): Wohnungsbaubericht Hamburg 2016, Hamburg, Deutschland, 2017c.

Frank, S. und Weck, S.: Being Good Parents or Being Good Citizens: Dilemmas and Contradictions of Urban Families in Middle-Class Enclaves and Mixed Neighbourhoods in Germany, Int. J. Urban Reg. Res., 42, 20-35, https://doi.org/10.1111/14682427.12592, 2018.

Habermas, J.: Legitimationsprobleme im Spätkapitalismus, Suhrkamp, Frankfurt am Main, Deutschland, 1973.

Haug, F.: Feministisch arbeiten mit Marx, UTOPIE kreativ, 109/110, 125-137, 1999.

Haug, F. und Hauser, K.: Marxistische Theorien und feministischer Standpunkt, in: TraditionenBrüche. Entwicklungen feministischer Theorie, Herausgeber: Knapp, G.-A. und Wetterer, A., Kore, Freiburg, Deutschland, 115-149, 1992.

Herrmann, U.: Hurra, wir dürfen zahlen. Der Selbstbetrug der Mittelschicht, Westend Verlag, Frankfurt am Main, Deutschland, 2010.

Holm, A.: Mehr Licht als Schatten. Berliner Wohnungspolitik in Rot-Rot-Grün, dérive, 68, 14-17, 2017.

Kemper, J. und Mösgen, A.: Die ungleiche Geographie des Elterngelds. Zur familienpolitischen Reproduktion gesellschaftlicher Ungleichheit, Sub/urban. Zeitschrift für kritische Stadtforschung, 5, 43-68, 2017.

Kuschinski, E.: Das Patriarchat aus dem Haus werfen. Interview mit Eva Kuschinski, online aufrufbar: https://kritisch-lesen.de/ interview/das-patriarchat-aus-dem-haus-werfen, letzter Zugriff: 11. April 2019.

Kuschinski, E. und Reichle, L. R.: Why housing is a feminist issue, oder warum die Wohnungsfrage feministisch zu stellen ist, Feministische Geo-RundMail, 81, 33-36, 2020.

Lebuhn, H., Holm, A., Junker, S., und Neitzel, K.: Wohnverhältnisse in Deutschland - eine Analyse der sozialen Lage in 77 Großstädten, Hans Böckler Stiftung, Berlin, Düsseldorf, Deutschland, 2017.

Menke, K. und Klammer, U.: Mehr Geschlechtergerechtigkeit - weniger soziale Gerechtigkeit? Familienpolitische Reformprozesse in Deutschland aus intersektionaler Perspektive, Sozialer Fortschritt, 66, 213-228, 2017.

Metzger, J. und Schipper, S.: Postneoliberale Strategien für bezahlbaren Wohnraum? Aktuelle wohnungspolitische Ansätze in Hamburg und Frankfurt am Main, in: Wohnraum für alle?! Perspektiven auf Planung, Politik und Architektur, Herausgeber: Schönig, B., Schipper, S., und Kadi, J., transcript, Bielefeld, Deutschland, 181-212, 2017.

Mieter helfen Mietern Hamburg, Diakonie Hamburg, Caritasverband für Hamburg e.V., und Stattbau Hamburg: Für eine neue soziale Wohnungspolitik in Hamburg, online aufrufbar: https://www.diakonie-hamburg. de/export/sites/default/.content/downloads/Presse/

Forderungen-fuer-eine-soziale-Wohnungspolitik-in-Hamburg Kopf-mit-vier-Logos_060716.pdf, letzter Zugriff: 12. Juli 2020.
O'Connor, J.: Die Finanzkrise des Staates, Suhrkamp, Frankfurt am Main, Deutschland, 1974.

Offe, C.: Strukturprobleme des kapitalistischen Staates, Suhrkamp, Frankfurt am Main, Deutschland, 1972.

Rinn, M.: Konflikte um die Stadt für alle - Das Machtfeld der Stadtentwicklungspolitik in Hamburg, Westfälisches Dampfboot, Münster, Deutschland, 2016.

Rinn, M.: Ein Urbanismus der Ungleichheit - „Neue soziale Stadtpolitik" in Hamburg als Strategie der Verbürgerlichung, Sub/urban. Zeitschrift für kritische Stadtforschung, 6, 9-28, 2018.

Schönig, B., Rink, D., Holm, A., und Gardemin, D.: Städte unter Druck. Die Rückkehr der Wohnungsfrage, Blätter für deutsche und internationale Politik, 2015, 69-79, 2015.

Schwiter, K., Berndt, C., und Truong, J.: Neoliberal austerity and the marketization of elderly care, Soc. Cult. Geogr., 19, 1-21, https://doi.org/10.1080/14649365.2015.1059473, 2015.

Siebel, W.: Stadt und soziale Ungleichheit, Leviathan, 40, 462-475, 2012.

Statistikamt Nord (Hrsg.): Hamburger Stadtteil-Profile 2016, Statistisches Amt für Hamburg und Schleswig-Holstein, Hamburg, Deutschland, 2018.

Statistikamt Nord (Hrsg.): Statistisches Jahrbuch Hamburg 2018/2019, Hamburg, Deutschland, 2019

Statistisches Bundesamt (Hrsg.): Kindertagesbetreuung regional 2007: Ein Vergleich aller 439 Kreise in Deutschland, Statistisches Bundesamt, Wiesbaden, Deutschland, 2008.

Statistisches Bundesamt (Hrsg.): Kindertagesbetreuung regional 2018. Ein Vergleich aller Kreise in Deutschland, Statistisches Bundesamt, Wiesbaden, Deutschland, online aufrufbar: http://www.statistikportal.de/sites/default/files/2019-07/ Kindertagesbetreuung_2018_barrierefrei.pdf (letzter Zugriff: 12. Juli 2020), 2019.

Statistisches Bundesamt: Tabelle A.7: Mediane und Armutsgefährdungsschwellen nach Regionen, online aufrufbar: https://www.statistikportal.de/de/sbe/ergebnisse/ einkommensarmut-und-verteilung/armutsgefaehrdung-5, letzter Zugriff: 12. Juli 2020.

Statistisches Landesamt Bremen, Statistisches Amt für Hamburg und Schleswig-Holstein, Statistisches Amt MecklenburgVorpommern, und Landesamt für Statistik Niedersachsen (Hrsg.): Metropolregion Hamburg - Endgültige Ergebnisse des Zensus vom 9. Mai 2011, Statistisches Amt für Hamburg und Schleswig-Holstein, Hamburg, Deutschland, 2016.

Vogelpohl, A. und Buchholz, T.: Breaking with Neoliberalization through Restricting the Housing Market: Novel urban policies and the case of Hamburg, Germany, Int. J. Urban Reg. Res., 41, 266-281, https://doi.org/10.1111/1468-2427.12490, 2017.

Vollmer, L. und Kadi, J.: Wohnungspolitik in der Krise des Neoliberalismus in Berlin und Wien. Postneoliberaler Paradigmenwechsel oder punktuelle staatliche Beruhigungspolitik?, Prokla, 48, 247-264, 2018.

Winker, G.: Überforderte Eltern zwischen Lohn- und Reproduktionsarbeit: Neoliberale Familienkonstruktionen ohne Zukunft, Z. Zeitschrift für marxistische Erneuerung, 103, 141-149, 2015. 